\title{
An in-depth stability analysis of nonuniform FDTD combined with novel local implicitization techniques
}

\author{
Arne Van Londersele, Daniël De Zutter, Dries Vande Ginste \\ Electromagnetics Group, Department of Information Technology, Ghent University/imec \\ Technologiepark 15, Gent, Belgium
}

\begin{abstract}
This work focuses on efficient full-wave solutions of multiscale electromagnetic problems in the time domain. Three local implicitization techniques are proposed and carefully analyzed in order to relax the traditional time step limit of the Finite-Difference Time-Domain (FDTD) method on a nonuniform, staggered, tensor product grid: Newmark, Crank-Nicolson (CN) and AlternatingDirection-Implicit (ADI) implicitization. All of them are applied in preferable directions, alike Hybrid Implicit-Explicit (HIE) methods, as to limit the rank of the sparse linear systems. Both exponential and linear stability are rigorously investigated for arbitrary grid spacings and arbitrary inhomogeneous, possibly lossy, isotropic media. Numerical examples confirm the conservation of energy inside a cavity for a million iterations if the time step is chosen below the proposed, relaxed limit. Apart from the theoretical contributions, new accomplishments such as the development of the leapfrog Alternating-Direction-HybridImplicit-Explicit (ADHIE) FDTD method and a less stringent Courant-like time step limit for the conventional, fully explicit FDTD method on a nonuniform grid, have immediate practical applications.

Keywords: Finite-Difference Time-Domain (FDTD), stability, nonuniform, Newmark, Crank-Nicolson (CN), Alternating-Direction-Implicit (ADI), Hybrid Implicit-Explicit (HIE), Alternating-Direction-Hybrid-Implicit-Explicit
\end{abstract} (ADHIE) 


\section{Introduction}

The Finite-Difference Time-Domain (FDTD) method is one of the prevalent numerical techniques to predict electromagnetic behavior by solving Maxwell's equations in the time domain. It discretizes the electric and magnetic fields on a cubic lattice in a staggered fashion such that the approximation of derivatives by central differences yields second-order accurate, explicit update equations. The algorithm marches on in time by alternately updating the electric and magnetic fields, which is also called leapfrog time stepping. This particular discretization, illustrated in Fig. 1, was pioneered by Kane Yee in 1966 [1]. Since then, the core of the algorithm has virtually remained unchanged and still persists in most commercial and academic FDTD packages. Often, the second-order accuracy is locally given up in favor of nonuniform grids, which preserve the tensor product nature but have step sizes that vary along the associated axis (e.g. $\Delta x$ varies along the $x$-axis). These nonuniform grids offer more flexibility, allowing to fit material boundaries with a smaller number of cells. One of the major drawbacks of FDTD, however, is the stability limit imposed on the time step, also known as the Courant limit, which for uniform grids reads

$$
\Delta t<\frac{1}{c_{0} \sqrt{\frac{1}{\Delta x^{2}}+\frac{1}{\Delta y^{2}}+\frac{1}{\Delta z^{2}}}},
$$

with $c_{0}$ the speed of light in vacuum. Various implicit and semi-implicit techniques have been proposed to sidestep (1) such that fewer time iterations are needed at the cost of more expensive computations per iteration. Generally, these techniques are applied throughout the whole grid because little is known about their influence on the stability limit if they would be applied locally.

The main goal of this paper is to put the stability of the FDTD method on nonuniform tensor product grids, as well as its combination with several local implicitization techniques, on a firm mathematical footing. Nowadays, with random memory access being far slower than the actual FDTD computations, direct matrix factorizations or iterative matrix inversions no longer pose a CPUtime limitation problem, especially if the occurring matrices are sparse and scale 


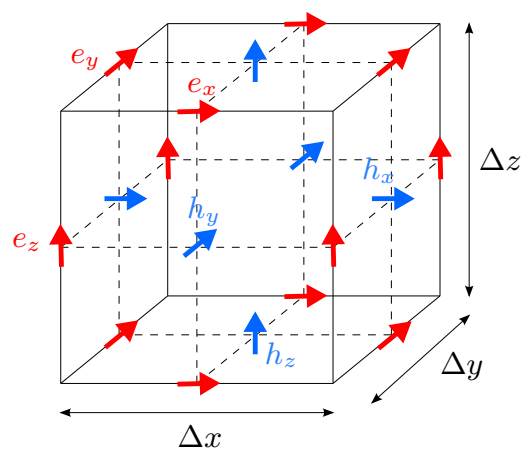

Figure 1: The traditional Yee cell. The electric field components (red) are discretized along the edges of the primary grid (full line). The magnetic field components (blue) are discretized along the edges of the dual grid (not shown). Electric and magnetic fields differ half a time step.

with only one out of three dimensions. The proposed techniques seek to meet this last requirement by allowing implicitization in preferable directions as to end up with low-rank, banded matrices. The typical configurations that will be tackled are illustrated in Fig. 2. If a thin layer or thin wire needs to be modeled, the nonuniform grid is locally much more dense. It will be demonstrated, both analytically and numerically, that the proposed local implicitization techniques allow to eliminate the small step sizes enforced by the thin object from the stability limit.

The remainder of this paper first lays out the update equations of each of the three proposed local implicitization techniques: Newmark, Crank-Nicolson $(\mathrm{CN})$ and Alternating-Direction-Implicit (ADI) implicitization. Next, in Section 3 , the stability of each technique is discussed. Two types of stability analyses are put forward: one based on $z$-transform theory and one extending the state-space-based method described in [2]. The Newmark implicitization technique is meticulously analyzed using the first approach. As a bonus, it leads to an explicit Courant-like time step limit for conventional FDTD on nonuniform grids that is less stringent than the one found in [3]. This new relaxed time step limit is directly applicable in classical FDTD software that leverages nonuni- 


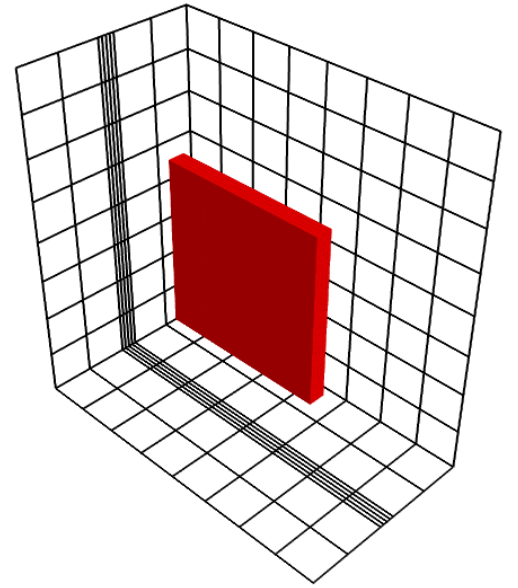

(a)

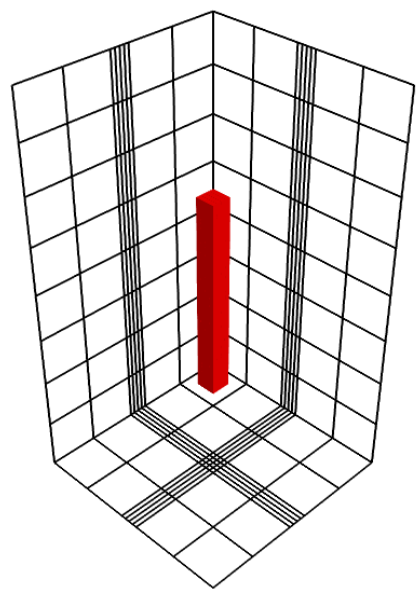

(b)

Figure 2: 3D representation of a nonuniform tensor product grid obtained by projecting the primary-grid edges on each of the three coordinate planes for (a) a thin plate and (b) a thin wire.

form gridding. The ADI implicitization technique is handled by the state-space approach, whereas the $\mathrm{CN}$ implicitization method is concisely analyzed using both approaches. In Section 4, the analytical results are numerically validated using the example of Fig. 2a. The paper concludes with a summary of the pros and cons of each local implicitization technique.

\section{Update equations}

\subsection{Continuous-time discrete-space system}

All proposed FDTD techniques use the same spatial discretization. More specifically, the electric and magnetic fields are discretized on a nonuniform tensor product grid comprising $n_{x} \times n_{y} \times n_{z}$ Yee cells. To investigate the stability under harsh conditions, the grid is terminated by perfectly electrically conducting (PEC) boundary conditions. The considered medium is isotropic, inhomogeneous and possibly lossy. The corresponding continuous-time discrete- 
space Maxwell equations are

$$
\begin{aligned}
D_{\varepsilon} \frac{\partial}{\partial t} e(t)+D_{\sigma_{e}} e(t)+j_{e}(t) & =\mathcal{C}_{h} h(t) \\
D_{\mu} \frac{\partial}{\partial t} h(t)+D_{\sigma_{m}} h(t)+j_{m}(t) & =-\mathcal{C}_{e} e(t)
\end{aligned}
$$

with row-major vectorized field unknowns and associated sources

$$
e=\left[\begin{array}{l}
e_{x} \\
e_{y} \\
e_{z}
\end{array}\right] \quad h=\left[\begin{array}{l}
h_{x} \\
h_{y} \\
h_{z}
\end{array}\right] \quad j_{e}=\left[\begin{array}{l}
j_{e, x} \\
j_{e, y} \\
j_{e, z}
\end{array}\right] \quad j_{m}=\left[\begin{array}{l}
j_{m, x} \\
j_{m, y} \\
j_{m, z}
\end{array}\right] .
$$

The total lengths of $e$ and $j_{e}$ are

$$
n_{e}=n_{x}\left(n_{y}-1\right)\left(n_{z}-1\right)+\left(n_{x}-1\right) n_{y}\left(n_{z}-1\right)+\left(n_{x}-1\right)\left(n_{y}-1\right) n_{z},
$$

whereas the total lengths of $h$ and $j_{m}$ are

$$
n_{h}=\left(n_{x}-1\right) n_{y} n_{z}+n_{x}\left(n_{y}-1\right) n_{z}+n_{x} n_{y}\left(n_{z}-1\right) .
$$

$D_{\varepsilon}, D_{\mu}, D_{\sigma_{e}}$ and $D_{\sigma_{m}}$ are the diagonal permittivity, permeability, electric conductivity and magnetic conductivity matrices respectively, which, from physical considerations, satisfy

$$
\begin{array}{rlrl}
{\left[D_{\varepsilon}\right]_{i i}>0} & {\left[D_{\sigma_{e}}\right]_{i i} \geq 0} & i=1, \ldots, n_{e} \\
{\left[D_{\mu}\right]_{j j}>0} & {\left[D_{\sigma_{m}}\right]_{j j} \geq 0} & j=1, \ldots, n_{h}
\end{array}
$$

The curl operators can be factorized as follows

$$
\mathcal{C}_{h}=\hat{\mathcal{V}} \hat{\mathcal{W}} \quad \mathcal{C}_{e}=\mathcal{V} \mathcal{C}^{T} \mathcal{W}
$$

with metric-free curl stencil $\mathcal{C} \in \mathbb{R}^{n_{e} \times n_{h}}$ given by

$$
\mathcal{C}=\left[\begin{array}{ccc}
0 & -I_{n_{x}} \otimes I_{n_{y}-1} \otimes \mathcal{D}_{n_{z}} & I_{n_{x}} \otimes \mathcal{D}_{n_{y}} \otimes I_{n_{z}-1} \\
I_{n_{x}-1} \otimes I_{n_{y}} \otimes \mathcal{D}_{n_{z}} & 0 & -\mathcal{D}_{n_{x}} \otimes I_{n_{y}} \otimes I_{n_{z}-1} \\
-I_{n_{x}-1} \otimes \mathcal{D}_{n_{y}} \otimes I_{n_{z}} & \mathcal{D}_{n_{x}} \otimes I_{n_{y}-1} \otimes I_{n_{z}} & 0
\end{array}\right],
$$

where ' $\otimes$ ' denotes the Kronecker product, $I_{n}$ the $n$-dimensional identity matrix, and $\mathcal{D}_{n_{u}} \in \mathbb{R}^{\left(n_{u}-1\right) \times n_{u}}$ the discrete differentiator defined as

$$
\mathcal{D}_{n_{u}}=\left[\begin{array}{ccccc}
-1 & 1 & & & \\
& -1 & 1 & & \\
& & \ddots & & \\
& & & -1 & 1
\end{array}\right]
$$


The metric of the grid is included in

$$
\begin{aligned}
& \mathcal{V}=\left[\begin{array}{lll}
I_{n_{x}-1} \otimes \delta_{y}^{-1} \otimes \delta_{z}^{-1} & & \\
& \delta_{x}^{-1} \otimes I_{n_{y}-1} \otimes \delta_{z}^{-1} & \\
& \delta_{x}^{-1} \otimes \delta_{y}^{-1} \otimes I_{n_{z}-1}
\end{array}\right] \\
& \mathcal{W}=\left[\begin{array}{lll}
\delta_{x} \otimes I_{n_{y}-1} \otimes I_{n_{z}-1} & & \\
& I_{n_{x}-1} \otimes \delta_{y} \otimes I_{n_{z}-1} & \\
& I_{n_{x}-1} \otimes I_{n_{y}-1} \otimes \delta_{z}
\end{array}\right] \\
& \hat{\mathcal{V}}=\left[\begin{array}{lll}
I_{n_{x}} \otimes \hat{\delta}_{y}^{-1} \otimes \hat{\delta}_{z}^{-1} & & \\
& \hat{\delta}_{x}^{-1} \otimes I_{n_{y}} \otimes \hat{\delta}_{z}^{-1} & \\
& \hat{\delta}_{x}^{-1} \otimes \hat{\delta}_{y}^{-1} \otimes I_{n_{z}}
\end{array}\right] \\
& \hat{\mathcal{W}}=\left[\begin{array}{lll}
\hat{\delta}_{x} \otimes I_{n_{y}} \otimes I_{n_{z}} & & \\
& I_{n_{x}} \otimes \hat{\delta}_{y} \otimes I_{n_{z}} & \\
& I_{n_{x}} \otimes I_{n_{y}} \otimes \hat{\delta}_{z}
\end{array}\right] \text {, }
\end{aligned}
$$

with $\delta_{u}$ and $\hat{\delta}_{u}$ diagonal matrices containing the $n_{u}$ and $n_{u}-1$ spatial increments in the $u$-dimension of the primary and dual grid respectively. Dual-grid operators are highlighted by a hat superscript. Recall that the electric and magnetic fields are discretized along primary- and dual-grid edges respectively.

\subsection{Discrete-time discrete-space system}

We summarize the update equations for each of the three implicitization techniques, where we choose to implicitize electric fields. A dual scheme, implicitizing the magnetic fields, of course, exists as well.

\subsubsection{Newmark implicitization}

The Newmark- $\beta$ technique is frequently used in Finite-Element Time-Domain (FETD) simulations (e.g. [4]), but is only recently investigated in the FDTD context. In one variant [5], both curls are time averaged as prescribed by the Newmark- $\beta$ scheme, whereas in another variant [6] only a single one is. Indeed, the unconditionally stable technique proposed in [6] is in fact the Newmark- $\beta$ time integration method with $\beta=0.25$, perceived as a dispersive background medium, which allows to interpret partial implicitization in one instead of two directions as a manifestation of anisotropy. Here, the unconditionally stable technique of [6] is extended to 3D and applied locally, which gives rise to the 
update scheme

$$
\begin{aligned}
& {\left.\left[\begin{array}{cc}
\frac{1}{\Delta t} D_{\varepsilon}+\frac{1}{2} D_{\sigma_{e}} & -\mathcal{C}_{h} \\
\frac{1}{4} \mathcal{C}_{e}\left(I_{n_{e}}-\mathcal{P}\right) & \frac{1}{\Delta t} D_{\mu}+\frac{1}{2} D_{\sigma_{m}}
\end{array}\right] x\right|^{n+2}} \\
& \quad=\left.\left[\begin{array}{cc}
\frac{1}{\Delta t} D_{\varepsilon}-\frac{1}{2} D_{\sigma_{e}} & 0 \\
-\frac{1}{2} \mathcal{C}_{e}\left(I_{n_{e}}+\mathcal{P}\right) & \frac{1}{\Delta t} D_{\mu}-\frac{1}{2} D_{\sigma_{m}}
\end{array}\right] x\right|^{n+1}+\left.\left[\begin{array}{cc}
0 & 0 \\
-\frac{1}{4} \mathcal{C}_{e}\left(I_{n_{e}}-\mathcal{P}\right) & 0
\end{array}\right] x\right|^{n}+\left.s\right|^{n+1}
\end{aligned}
$$

with

$$
\left.x\right|^{n}=\left.\left[\begin{array}{c}
e(n \Delta t) \\
h((n-0.5) \Delta t)
\end{array}\right] \quad s\right|^{n}=\left[\begin{array}{c}
j_{e}(n \Delta t) \\
j_{m}((n-0.5) \Delta t)
\end{array}\right]
$$

and $\mathcal{P}$ the $n_{e}$-dimensional diagonal matrix with elements

$$
[\mathcal{P}]_{i, i}=\left\{\begin{array}{ll}
1 & \text { if } e_{i} \text { is updated explicitly } \\
0 & \text { if } e_{i} \text { is updated implicitly }
\end{array} .\right.
$$

\section{3. $C N$ implicitization}

The Crank-Nicolson (CN) method is well-known in the FDTD community (e.g. [7]). If applied locally, its update scheme is

$$
\left.\left[\begin{array}{cc}
\frac{1}{\Delta t} D_{\varepsilon}+\frac{1}{2} D_{\sigma_{e}} & -\frac{1}{2}\left(I_{n_{e}}+\mathcal{P}\right) \mathcal{C}_{h} \\
\frac{1}{2} \mathcal{C}_{e}\left(I_{n_{e}}-\mathcal{P}\right) & \frac{1}{\Delta t} D_{\mu}+\frac{1}{2} D_{\sigma_{m}}
\end{array}\right] x\right|^{n+1}=\left.\left[\begin{array}{cc}
\frac{1}{\Delta t} D_{\varepsilon}-\frac{1}{2} D_{\sigma_{e}} & \frac{1}{2}\left(I_{n_{e}}-\mathcal{P}\right) \mathcal{C}_{h} \\
-\frac{1}{2} \mathcal{C}_{e}\left(I_{n_{e}}+\mathcal{P}\right) & \frac{1}{\Delta t} D_{\mu}-\frac{1}{2} D_{\sigma_{m}}
\end{array}\right] x\right|^{n}+\left.s\right|^{n}
$$

with

$$
\left.x\right|^{n}=\left[\begin{array}{c}
\mathcal{P} e(n \Delta t)+\left(I_{n_{e}}-\mathcal{P}\right) e((n-0.5) \Delta t) \\
h((n-0.5) \Delta t)
\end{array}\right]
$$

and $\mathcal{P}$ given by (18). The time discretization of the implicitized electric fields is identical to that of the magnetic fields. A similar time discretization holds for the sources.

\subsection{ADI implicitization}

Although the original Alternating-Direction-Implicit (ADI) FDTD method was a split-step method where additional field variables were needed at intermediate time instances, it has evolved to a one-step leapfrog update scheme with the same dispersion and stability properties but improved computation time and memory requirements [8]. Both the split-step and the leapfrog ADI 
method split the entire curl into two parts, e.g. $\mathcal{C}_{e}=\mathcal{C}_{e}^{1}+\mathcal{C}_{e}^{2}$ for the electricfield curl, and this in a clever way as to end up with tridiagonal matrices that scale with the number of cells in only one dimension (either $n_{x}, n_{y}$ or $n_{z}$ ). In the remainder of this manuscript, we will refer to this as complete curl splitting as opposed to incomplete curl splitting, which will be presented here for the first time (see Section 3.3.2). Our new curl splitting technique generalizes the traditional leapfrog ADI method, which is fully implicit and unconditionally stable, to a so-called Hybrid Implicit-Explicit (HIE) ADI method, named "leapfrog ADHIE-FDTD method", which is only partially implicit and weakly conditionally stable. Without going into the details yet, both the traditional leapfrog ADI and the newly proposed leapfrog ADHIE update scheme can be cast as:

$$
\begin{aligned}
& {\left.\left[\begin{array}{cc}
\frac{1}{\Delta t} D_{\varepsilon}+\frac{1}{2} D_{\sigma_{e}}+\frac{\Delta t}{4 \alpha^{2}} \mathcal{C}_{h 1} D_{\mu}^{-1} \mathcal{C}_{e 1} & -\mathcal{C}_{h} \\
0 & \frac{1}{\Delta t} D_{\mu}+\frac{1}{2} D_{\sigma_{m}}+\frac{\Delta t}{4 \alpha^{2}} \mathcal{C}_{e 2} D_{\varepsilon}^{-1} \mathcal{C}_{h 2}
\end{array}\right] x\right|^{n+1}} \\
& \quad=\left.\left[\begin{array}{cc}
\frac{1}{\Delta t} D_{\varepsilon}-\frac{1}{2} D_{\sigma_{e}}+\frac{\Delta t}{4 \alpha^{2}} \mathcal{C}_{h 1} D_{\mu}^{-1} \mathcal{C}_{e 1} & 0 \\
-\mathcal{C}_{e} & \frac{1}{\Delta t} D_{\mu}-\frac{1}{2} D_{\sigma_{m}}+\frac{\Delta t}{4 \alpha^{2}} \mathcal{C}_{e 2} D_{\varepsilon}^{-1} \mathcal{C}_{h 2}
\end{array}\right] x\right|^{n}+\left.s\right|^{n}
\end{aligned}
$$

where $\left.x\right|^{n}$ and $\left.s\right|^{n}$ are given by (17). The scalar $\alpha$ is a tunable parameter that has to be chosen in the interval $] 0,1[$ for incomplete curl splitting and equals one for complete curl splitting. The curl parts $\mathcal{C}_{e 1}, \mathcal{C}_{h 1}, \mathcal{C}_{e 2}$ and $\mathcal{C}_{h 2}$ will be defined in Section 3.3. Note that, if they were zero, (21) would reduce to the conventional, fully explicit FDTD method. Hence, they constitute a perturbation, which results in the so-called ADI splitting error [9, eq. 11]. This leads to non-negligible numerical errors for time steps considerably exceeding the Courant limit (1) and for electromagnetic fields with large spatial gradients. 


\section{Stability}

\subsection{Newmark implicitization}

\subsubsection{Exponential stability}

The discrete-time system (16) is transformed to the $z$-domain, for the most delicate case where all energy is trapped inside a lossless cavity, resulting in the linear system

$$
A x-s=0,
$$

where

$$
x=\left.\sum_{n=0}^{\infty} x\right|^{n} z^{-n} \quad s=\left.\sum_{n=0}^{\infty} s\right|^{n} z^{-n}
$$

with initial conditions

$$
\left.x\right|^{0}=\left.x\right|^{1}=\left.s\right|^{0}=0
$$

and system matrix

$$
A=\left[\begin{array}{cc}
(z-1) \Delta t^{-1} D_{\varepsilon} & -z(\hat{\mathcal{V}} \mathcal{\mathcal { W }}) \\
\left(\mathcal{V} \mathcal{C}^{T} \mathcal{W}\right) \mathcal{I} & (z-1) \Delta t^{-1} D_{\mu}
\end{array}\right]
$$

The diagonal matrix $\mathcal{I} \in \mathbb{C}^{n_{e} \times n_{e}}$, occurring in (25), has elements

$$
[\mathcal{I}]_{i, i}= \begin{cases}1 & \text { if } e_{i} \text { is updated explicitly } \\ (z+1)^{2} /(4 z) & \text { if } e_{i} \text { is updated implicitly }\end{cases}
$$

Exponentially growing instabilities are excluded if the poles of the transfer function matrix belonging to (22), which are the solutions for $z$ fulfilling $\operatorname{det}(A)=0$, do not lie outside the unit disk [10]. Note that these solutions do not change upon replacement of $A$ with

$$
\tilde{A}=Q^{-1} R^{-1} A Q=\left[\begin{array}{cc}
(z-1) \Delta t^{-1} I_{n_{e}} & -z \tilde{\mathcal{C}} \\
\tilde{\mathcal{C}}^{T} \mathcal{I} & (z-1) \Delta t^{-1} I_{n_{h}}
\end{array}\right]
$$

with regular matrices

$$
R=\left[\begin{array}{cc}
D_{\varepsilon} & \\
& D_{\mu}
\end{array}\right] \quad Q=\left[\begin{array}{ll}
D_{\varepsilon}^{-1 / 2} \hat{\mathcal{V}}^{1 / 2} \mathcal{W}^{-1 / 2} & \\
& \\
& D_{\mu}^{-1 / 2} \mathcal{V}^{1 / 2} \hat{\mathcal{W}}^{-1 / 2}
\end{array}\right],
$$

and modified curl operator

$$
\tilde{\mathcal{C}}=D_{\varepsilon}^{-1 / 2} \hat{\mathcal{V}}^{1 / 2} \mathcal{W}^{1 / 2} \mathcal{C} D_{\mu}^{-1 / 2} \mathcal{V}^{1 / 2} \hat{\mathcal{W}}^{1 / 2}
$$


We used the fact that all matrices occurring in (28) are diagonal and regular, and that $\mathcal{I}$ is diagonal as well. The remainder of this stability analysis focuses on the matrix $\tilde{A}$, which has "more symmetry" than the original matrix $A$.

Similarly to $[4,11]$, we need to distinguish between the static modes with $z=1$ and the dynamic modes with $z \neq 1$. The former obviously have unitcircle-bounded $z$ and reside in the null space of the system matrix (25) with insertion of $z=1$. Using the symmetry-introducing transformation (27), they can also be found upon inspection of the nullspace of

$$
\tilde{A}_{z=1}=\left[\begin{array}{cc}
0 & -\tilde{\mathcal{C}} \\
\tilde{\mathcal{C}}^{T} & 0
\end{array}\right]
$$

For the dynamic modes, the block matrices on the diagonal of (27) are nonsingular and the stability analysis amounts to locating the roots of

$$
\begin{aligned}
& \operatorname{det}\left(\tilde{A}_{z \neq 1}\right) \\
& =\operatorname{det}\left((z-1) \Delta t^{-1} I_{n_{e}}\right) \operatorname{det}\left((z-1) \Delta t^{-1} I_{n_{h}}+z(z-1)^{-1} \Delta t \tilde{\mathcal{C}}^{T} \mathcal{I} \tilde{\mathcal{C}}\right) \\
& =\left((z-1) \Delta t^{-1}\right)^{n_{e}+n_{h}} \operatorname{det}\left(I_{n_{h}}+z(z-1)^{-2} \Delta t^{2} \tilde{\mathcal{C}}^{T} \mathcal{I} \tilde{\mathcal{C}}\right)
\end{aligned}
$$

where all roots are those of the remaining determinant. Along the lines of [12], substituting the bilinear transformation

$$
z=\frac{\zeta-1}{\zeta+1}
$$

which projects the unit disk in $z$-space to the right half-plane in $\zeta$-space, yields

$$
\operatorname{det}\left(I_{n_{h}}+\frac{\Delta t^{2}}{4} \tilde{\mathcal{C}}^{T}\left(\zeta^{2} I_{n_{e}}-\mathcal{P}\right) \tilde{\mathcal{C}}\right)=0
$$

with $\mathcal{P}$ given by (18). Searching the roots of (33) is equivalent to solving the generalized eigenvalue problem

$$
\zeta^{2} \tilde{\mathcal{C}}^{T} \tilde{\mathcal{C}} v=\left(\tilde{\mathcal{C}}^{T} \mathcal{P} \tilde{\mathcal{C}}-4 \Delta t^{-2} I_{n_{h}}\right) v
$$

The zero eigenvalues of the curl-curl matrix in the l.h.s. of (34) give rise to so-called infinite eigenvalues $\zeta^{2}=\infty$ of the generalized eigenvalue problem, which are doubly mapped to $z=1$. They correspond to static modes, which 
have already been treated above and which were not allowed in the derivation of (31). Therefore, we only consider the finite eigenvalues of (34), which correspond to the dynamic modes, and we determine their location in the complex plane. Left-multiplying (34) by the hermitian transpose of $v$ and subsequently subtracting/adding the hermitian-transposed equation, yields respectively

$$
\begin{array}{ll}
\operatorname{Im}\left(\zeta^{2}\right)\|\tilde{\mathcal{C}} v\|_{2}^{2}=0 & \forall v \neq 0 \\
\operatorname{Re}\left(\zeta^{2}\right)\|\tilde{\mathcal{C}} v\|_{2}^{2}=\|\mathcal{P} \tilde{\mathcal{C}} v\|_{2}^{2}-4 \Delta t^{-2}\|v\|_{2}^{2} & \forall v \neq 0
\end{array}
$$

where we readily replaced the occurring inner products by vector 2-norms and used the fact that $\mathcal{P}=\mathcal{P}^{T} \mathcal{P}$. Now, recall that exponentially growing instabilities are excluded by demanding that $|z| \leq 1$, which is, via the bilinear transformation (32), translated to $\operatorname{Re}(\zeta) \geq 0$. From (35), we conclude that $\operatorname{Im}\left(\zeta^{2}\right)=0$, such that any solution $\zeta^{2}$ yields either two real or two purely imaginary values for $\pm \zeta$. Since the first scenario always implies that one of the two values $\pm \zeta$ resides on the negative real axis, the only way both $\pm \zeta$ satisfy $\operatorname{Re}(\zeta) \geq 0$ is the case where they are purely imaginary. These purely imaginary values for $\pm \zeta$ yield two complex conjugate roots $z$ lying on the unit circle. From a physical perspective, this is exactly what we expect from a passive, lossless electromagnetic system; no energy is created, nor is there energy lost. In conclusion, stability is guaranteed if $\operatorname{Re}\left(\zeta^{2}\right) \leq 0$, which is satisfied if and only if the r.h.s. of (36) cannot become positive. This determines an upper bound on the time step

$$
\Delta t \leq \frac{2}{\|\mathcal{P} \tilde{\mathcal{C}}\|_{2}},
$$

with the matrix 2-norm defined as [13, p. 476]

$$
\|\mathcal{P} \tilde{\mathcal{C}}\|_{2}=\max _{v \neq 0} \frac{\|\mathcal{P} \tilde{\mathcal{C}} v\|_{2}}{\|v\|_{2}}=\sigma_{\max }(\mathcal{P} \tilde{\mathcal{C}})=\sqrt{\lambda_{\max }\left(\tilde{\mathcal{C}}^{T} \mathcal{P} \tilde{\mathcal{C}}\right)}
$$

The functions $\sigma_{\max }()$ and $\lambda_{\max }()$ denote the largest singular value and eigenvalue respectively. For a uniform grid that is homogeneously filled with vacuum and for which we fully explicitly advance in time, i.e. $\mathcal{P}=I_{n_{e}}$, this upper bound is identical to the one found in [2], apart from the strict inequality. In Section 3.1.4, it will be shown that thoughtful implicitization, which eliminates 
all small spatial steps beneath a certain threshold from the r.h.s. of (37) by selecting the pertinent $[\mathcal{P}]_{i, i}$ to be zero, can considerably reduce the time step limit for configurations with strongly varying spatial step sizes.

\subsubsection{Linear stability}

Up till now, we only discussed exponential stability, meaning that the fields cannot diverge exponentially, which is achieved by the condition $|z| \leq 1$. As pointed out in [14], however, unit circle boundedness of $z$ does not fully guarantee Lyapunov stability [13, p. 670], which simply means that the fields cannot diverge to infinity in any kind of fashion (exponentially, polynomially,...). In addition, the poles located on the unit circle should be semisimple. In other words, they should have equal algebraic and geometric multiplicities [15, p.296], which is identical to the corresponding eigenvectors being linearly independent or, equivalently, to the corresponding matrix being diagonalizable. For a two-

level update scheme $\left.x\right|^{n+1}=\left.M x\right|^{n}$, a violation of this condition manifests itself in a subtle linear growth of $x$ because the amplification matrix $M$ then has at least one Jordan block of the form

$$
\left[\begin{array}{ll}
\lambda & 1 \\
0 & \lambda
\end{array}\right]
$$

with $|\lambda|=1$, which is clearly unbounded upon self-multiplication. The same reasoning holds for higher-level schemes, as it is always possible to reduce them to a two-level scheme via a proper change of variables, e.g. companion linearization. In [4], the FETD method with Newmark- $\beta$ time integration of the second-order wave equation is shown to be prone to this type of late-time instability.

We will show now that our proposed Newmark implicitization method, and by extension also the conventional FDTD method, is free of linear instabilities. Therefore, note that there are $2\left(n_{e}+n_{h}\right)$ poles $z$ that satisfy $\operatorname{det}(A)=0$, which can be essentially categorized into four types:

- complex conjugate pole pairs $\left(z, z^{*}\right)$ with $|z|=1$, yielding dynamic modes

- the repeated pole $z=1$, yielding static modes 
- the repeated pole $z=-1$, yielding "almost unstable" dynamic modes

- the non-physical repeated pole $z=0$

First, we discuss the last category, which in our scheme only appears as an algebraic artefact due to the linearization of the three-level Newmark scheme (16). If we shortly write (16) as

$$
\left.M_{2} x\right|^{n+2}=\left.M_{1} x\right|^{n+1}+\left.M_{0} x\right|^{n}+s^{n+1},
$$

then the companion linearization is

$$
\left[\begin{array}{cc}
M_{2} & 0 \\
0 & I_{n_{e}+n_{h}}
\end{array}\right]\left[\begin{array}{l}
\left.x\right|^{n+2} \\
\left.x\right|^{n+1}
\end{array}\right]=\left[\begin{array}{cc}
M_{1} & M_{0} \\
I_{n_{e}+n_{h}} & 0
\end{array}\right]\left[\begin{array}{c}
\left.x\right|^{n+1} \\
\left.x\right|^{n}
\end{array}\right]+\left[\begin{array}{c}
\left.s\right|^{n+1} \\
0
\end{array}\right] .
$$

The block structure of the matrix in the r.h.s. of (41) is given by

$$
\left[\begin{array}{cccc}
B_{n_{e} \times n_{e}} & 0 & 0 & 0 \\
B_{n_{h} \times n_{e}} & B_{n_{h} \times n_{h}} & B_{n_{h} \times n_{e}} & 0 \\
B_{n_{e} \times n_{e}} & 0 & 0 & 0 \\
0 & B_{n_{h} \times n_{h}} & 0 & 0
\end{array}\right],
$$

where $B_{n_{1} \times n_{2}}$ denotes an arbitrary block of size $n_{1} \times n_{2}$ that has at least one non-zero element. A block permutation of the second and third row followed by a block permutation of the second and third column reveals that this matrix has at least $n_{e}+n_{h}$ zero eigenvalues or, equivalently, that the transfer function has a pole $z=0$ with multiplicity $n_{e}+n_{h}$ or more. This pole would also have been found in the analysis of Section 3.1.1, if the source in (16) was shifted one step back in time; $\left.s\right|^{n}$ instead of $\left.s\right|^{n+1}$. Anyway, since $z=0$ lies inside the unit disk, it is harmless. However, this zero pole, which originates from the sparsity of the matrix $M_{0}$, poses a fundamental difference between our proposed Newmark implicitization technique and the Newmark FETD method [4], because the linearized system (41) only requires $n_{e}+n_{h}$ instead of $2\left(n_{e}+n_{h}\right)$ linearly independent modes. For this reason, it is very likely that the Newmark- $\beta$ FDTD method described in [5] suffers from linearly growing non-physical fields analogous to [4].

We will now prove that the remaining $n_{e}+n_{h}$ poles belong to one of the above three mentioned categories on the unit circle and we will propose a sufficient condition such that they are guaranteed to be semisimple. The poles 
from the first category give rise to linearly independent dynamic modes in the $\zeta^{2}$-domain due to Lemma 1 (see Appendix). Since every $\zeta^{2}$ is, in a one-to-one sense, mapped to a complex conjugate pair $\left(z, z^{*}\right)$ with $z \neq z^{*}$, this linear independence is preserved in the $z$-domain. Thus, the first category of poles is semisimple. A similar reasoning could be repeated for the second category, but since $z=z^{*}=-1$, linear independence of the dynamic modes in the $z$-domain cannot be guaranteed. This is, however, easily remedied by imposing a strict inequality

$$
\Delta t<\frac{2}{\|\mathcal{P} \tilde{\mathcal{C}}\|_{2}}
$$

instead of (37). Now, we must show that the third category of poles, corresponding to the static modes, is semisimple. Since these modes are known to span the nullspace of the skew-symmetric matrix (30), the number of linearly independent static modes is given by

$$
n_{\text {stat }}=\operatorname{null}(\tilde{\mathcal{C}})+\operatorname{null}\left(\tilde{\mathcal{C}}^{T}\right)
$$

where null() denotes the nullity, i.e. the dimension of the nullspace. It now suffices to show that these static modes together with the dynamic modes span the entire space $\mathbb{R}^{\left(n_{e}+n_{h}\right) \times\left(n_{e}+n_{h}\right)}$. From (34), it is found that the number of dynamic modes is

$$
n_{\mathrm{dyn}}=2\left(n_{h}-\operatorname{null}\left(\tilde{\mathcal{C}}^{T} \tilde{\mathcal{C}}\right)\right)
$$

Since from [15, p. 96],

$$
\operatorname{null}\left(\tilde{\mathcal{C}}^{T} \tilde{\mathcal{C}}\right)=\operatorname{null}(\tilde{\mathcal{C}})
$$

we obtain

$$
n_{\mathrm{stat}}+n_{\mathrm{dyn}}=2 n_{h}-\operatorname{null}(\tilde{\mathcal{C}})+\operatorname{null}\left(\tilde{\mathcal{C}}^{T}\right)=n_{e}+n_{h},
$$

where the last equality is indeed true because, e.g. for the given PEC boundary conditions, which imply that $n_{h}>n_{e}$, we have

$$
\operatorname{null}(\tilde{\mathcal{C}})=n_{h}-n_{e}+\operatorname{null}\left(\tilde{\mathcal{C}}^{T}\right) .
$$

In conclusion, Lyapunov stability, which encompasses both linear and exponential stability, is guaranteed if the time step satisfies (43). For uniform grids with 
explicit time stepping $\left(\mathcal{P}=I_{n_{e}}\right)$, this agrees with [16], where the strict inequality (43) is found to be a necessary and sufficient condition for Lyapunov stability, and agrees with [17], where the linear instability is numerically observed for a one-dimensional conventional FDTD simulation running at the "magic time step", i.e. , corresponding to an equality sign in (43).

As a last remark, note that for a fully implicit grid $(\mathcal{P}=0)$ holds that $\operatorname{det}\left(\tilde{A}_{z=-1}\right) \neq 0$, such that the Newmark FDTD method (16) is free of linear instability.

\subsubsection{A Courant-like stability limit for fully explicit, nonuniform grids}

To obtain a more transparent expression for the maximum allowed time step, we first derive an upper bound for the denominator of (43) in the simplified case that $\mathcal{P}=I_{n_{e}}$. In other words, we consider a conventional FDTD scheme with explicit leapfrog time stepping in each point of the grid. It is helpful to introduce the matrix

$$
\mathcal{K}=\left[\begin{array}{ccc}
0 & -I_{n_{x}} \otimes I_{n_{y}-1} \otimes \mathcal{K}_{z} & I_{n_{x}} \otimes \mathcal{K}_{y} \otimes I_{n_{z}-1} \\
I_{n_{x}-1} \otimes I_{n_{y}} \otimes \mathcal{K}_{z} & 0 & -\mathcal{K}_{x} \otimes I_{n_{y}} \otimes I_{n_{z}-1} \\
-I_{n_{x}-1} \otimes \mathcal{K}_{y} \otimes I_{n_{z}} & \mathcal{K}_{x} \otimes I_{n_{y}-1} \otimes I_{n_{z}} & 0
\end{array}\right],
$$

with

$$
\mathcal{K}_{u}=\hat{\delta}_{u}^{-1 / 2} \mathcal{D}_{n_{u}} \delta_{u}^{-1 / 2}
$$

As proven in [2, eq. 44], a matrix of this particular form has the property

$$
\|\mathcal{K}\|_{2}^{2}=\left\|\mathcal{K}_{x}\right\|_{2}^{2}+\left\|\mathcal{K}_{y}\right\|_{2}^{2}+\left\|\mathcal{K}_{z}\right\|_{2}^{2} .
$$

Now note that

$$
\tilde{\mathcal{C}}=D_{\varepsilon}^{-1 / 2} \mathcal{K} D_{\mu}^{-1 / 2}
$$

Using submultiplicativity of the matrix 2-norm [15, p. 550], yields

$$
\|\tilde{\mathcal{C}}\|_{2}^{2} \leq \frac{\|\mathcal{K}\|_{2}^{2}}{\varepsilon^{\min } \mu^{\min }}
$$

with $\varepsilon^{\min }$ and $\mu^{\min }$ the lowest permittivity and permeability among all cells, which are often those of vacuum. A similar reasoning shows that

$$
\left\|\mathcal{K}_{u}\right\|_{2}^{2} \leq \frac{\left\|\mathcal{D}_{n_{u}}\right\|_{2}^{2}}{\delta_{u}^{\min } \hat{\delta}_{u}^{\min }}
$$


with $\delta_{u}^{\min }$ and $\hat{\delta}_{u}^{\min }$ the smallest spatial $u$-step present in the primary and dual grid respectively, and [2, eq. 50]

$$
\left\|\mathcal{D}_{n_{u}}\right\|_{2}=2 \cos \left(\frac{\pi}{2 n_{u}}\right) .
$$

So, if no implicitization is applied, (51) together with (53)-(55) substituted into (43) yields the time step limit

$$
\Delta t<\frac{1}{\sqrt{\frac{1}{\varepsilon^{\min } \mu^{\min }}}} \frac{1}{\sqrt{\frac{\cos ^{2}\left(\frac{\pi}{2 n x}\right)}{\delta_{x}^{\min \delta_{x}^{\min }}}+\frac{\cos ^{2}\left(\frac{\pi}{2 n y}\right)}{\delta_{y}^{\min } \hat{\delta}_{y}^{\min }}+\frac{\cos ^{2}\left(\frac{\pi}{2 n z}\right)}{\delta_{z}^{\min \delta_{z}^{\min }}}}},
$$

which is a generalization of the conventional Courant limit to nonuniform grids. Note that (56) provides a tighter upper bound than the one proposed in [3].

\subsubsection{Local implicitization to relax the stability limit}

Now, we elaborate on how local implicitization affects the explicit stability limit (56) and, more importantly, how $\mathcal{P}$ can be tuned to get a more loose constraint. Suppose $\hat{\delta}_{x}^{\min }=\hat{\delta}_{x, i}$, then we could implicitize all $e_{y}$ and $e_{z}$ with $x$-index $i$, which corresponds to

$$
\mathcal{P}=\left[\begin{array}{lll}
I_{n_{x}} \otimes I_{n_{y}-1} \otimes I_{n_{z}-1} & & \\
& \mathcal{P}_{n_{x}-1}^{i} \otimes I_{n_{y}} \otimes I_{n_{z}-1} & \\
& & \mathcal{P}_{n_{x}-1}^{i} \otimes I_{n_{y}-1} \otimes I_{n_{z}}
\end{array}\right],
$$

where we used the notation $\mathcal{P}_{n}^{k}$ to denote the diagonal projection matrix that is constructed by setting the $k^{\text {th }}$ element of the $n$-dimensional identity matrix to zero. Now, (53) changes to

$$
\|\mathcal{P} \tilde{\mathcal{C}}\|_{2}^{2} \leq \frac{\|\mathcal{P} \mathcal{K}\|_{2}^{2}}{\varepsilon^{\min } \mu^{\min }}
$$

Because of the specific structure of $\mathcal{P}$, essentially being a projection matrix, one can see that

$$
\mathcal{P} \mathcal{K}=\mathcal{P}\left[\begin{array}{ccc}
0 & -I_{n_{x}} \otimes I_{n_{y}-1} \otimes \mathcal{K}_{z} & I_{n_{x}} \otimes \mathcal{K}_{y} \otimes I_{n_{z}-1} \\
I_{n_{x}-1} \otimes I_{n_{y}} \otimes \mathcal{K}_{z} & 0 & -\mathcal{P}_{n_{x}-1}^{i} \mathcal{K}_{x} \otimes I_{n_{y}} \otimes I_{n_{z}-1} \\
-I_{n_{x}-1} \otimes \mathcal{K}_{y} \otimes I_{n_{z}} & \mathcal{P}_{n_{x}-1}^{i} \mathcal{K}_{x} \otimes I_{n_{y}-1} \otimes I_{n_{z}} & 0
\end{array}\right]
$$

Consequently, submultiplicativity implies that (51) gives rise to

$$
\|\mathcal{P} \mathcal{K}\|_{2}^{2} \leq\left\|\mathcal{P}_{n_{x}-1}^{i} \mathcal{K}_{x}\right\|_{2}^{2}+\left\|\mathcal{K}_{y}\right\|_{2}^{2}+\left\|\mathcal{K}_{z}\right\|_{2}^{2}
$$




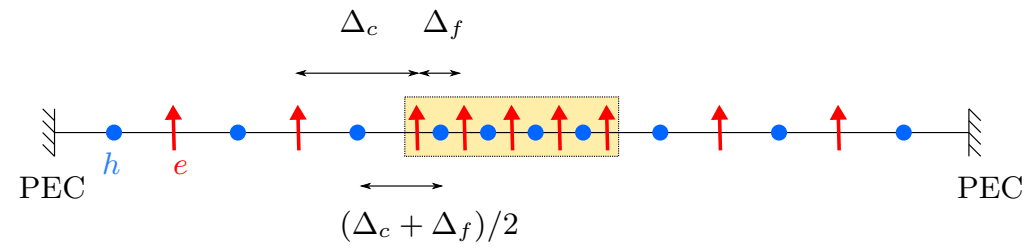

Figure 3: A one-dimensional refinement scheme with coarse step $\Delta_{c}$, fine step $\Delta_{f}$ and average-sized transition step. The implicitized fields are highlighted by a yellow box. The Courant limit solely depends on $\Delta_{c}$.

Since $\mathcal{P}_{n_{x}-1}^{i}$ directly acts on $\hat{\delta}_{x}^{-1 / 2}$, we succeeded in eliminating $\hat{\delta}_{x, i}$ from the stability limit, and $\hat{\delta}_{x}^{\min }$ in (56) is replaced by the smallest step amongst those that were not eliminated via implicitization. If we would like to eliminate $\delta_{x, i}$, it suffices to implicitize all $e_{y}$ and $e_{z}$ with $x$-index $i-1$ and $i$, because $\mathcal{D}_{n_{x}}$ is a bidiagonal matrix. This means that the one-dimensional refinement scheme illustrated in Fig. 3 is stable under the Courant limit imposed by the coarse step $\Delta_{c}$, if the yellow-boxed fields are implicitly updated.

\subsection{CN implicitization}

The conditional stability of the local application of Crank-Nicolson time integration will be proven in two different ways. The first one is inspired by the previous analysis for Newmark implicitization. The second one is built on the insights of [2]. It forms the bridge to the third and last implicitization technique which locally applies the leapfrog ADI method in preferable directions. Both arrive at the same conclusion.

\subsubsection{First stability analysis}

The $z$-transform of the discrete-time system (19), again in the lossless case, yields the system matrix

$$
A_{\mathrm{cn}}=\left[\begin{array}{cc}
(z-1) \Delta t^{-1} D_{\varepsilon} & -z \mathcal{I}_{2}(\hat{\mathcal{V}} \mathcal{C} \hat{\mathcal{W}}) \\
\left(\mathcal{V} \mathcal{C}^{T} \mathcal{W}\right) \mathcal{I}_{1} & (z-1) \Delta t^{-1} D_{\mu}
\end{array}\right],
$$


where

$$
\left[\mathcal{I}_{1}\right]_{i, i}= \begin{cases}1 & {\left[\mathcal{I}_{2}\right]_{i, i}=\left\{\begin{array}{ll}
1 & \text { if } e_{i} \text { is updated explicitly } \\
(z+1) / 2
\end{array} \quad \text { if } e_{i}\right. \text { is updated implicitly }}\end{cases}
$$

The steps in Section 3.1.1 that led to (31), can be repeated yielding the characteristic equation

$$
\operatorname{det}\left(I_{n_{h}}+z(z-1)^{-2} \Delta t^{2} \tilde{\mathcal{C}}^{T} \mathcal{I}_{1} \mathcal{I}_{2} \tilde{\mathcal{C}}\right)=0 .
$$

Somewhat surprisingly, this characteristic equation is identical to the one of Newmark implicitization since $\mathcal{I}_{1} \mathcal{I}_{2}=\mathcal{I}$. Hence, the exact same conclusions can be drawn for the relaxation of the Courant limit using CN implicitization as was the case for Newmark implicitization.

\subsubsection{Second stability analysis}

Using $R$ and $Q$ as defined in (28), we apply the symmetry-introducing transformation as in (27) to (19), including losses but omitting the source term, which yields

$$
\left.\left(E_{\mathrm{cn}}+F_{\mathrm{cn}}\right) \tilde{x}\right|^{n+1}=\left.\left(E_{\mathrm{cn}}-F_{\mathrm{cn}}\right) \tilde{x}\right|^{n},
$$

with decomposed update matrices

$$
E_{\mathrm{cn}}=\left[\begin{array}{cc}
\frac{1}{\Delta t} I_{n_{e}} & -\frac{1}{2} \mathcal{P} \tilde{\mathcal{C}} \\
-\frac{1}{2} \tilde{\mathcal{C}}^{T} \mathcal{P} & \frac{1}{\Delta t} I_{n_{h}}
\end{array}\right] \quad F_{\mathrm{cn}}=\left[\begin{array}{cc}
\frac{1}{2} D_{\varepsilon}^{-1} D_{\sigma_{e}} & -\frac{1}{2} \tilde{\mathcal{C}} \\
\frac{1}{2} \tilde{\mathcal{C}}^{T} & \frac{1}{2} D_{\mu}^{-1} D_{\sigma_{m}}
\end{array}\right]
$$

rescaled field vectors

$$
\left.\tilde{x}\right|^{n}=\left[\begin{array}{c}
\left.D_{\varepsilon}^{1 / 2} \hat{\mathcal{V}}^{-1 / 2} \mathcal{W}^{1 / 2} e\right|^{n} \\
\left.D_{\mu}^{1 / 2} \mathcal{V}^{-1 / 2} \hat{\mathcal{W}}^{1 / 2} h\right|^{n}
\end{array}\right]=\left[\begin{array}{c}
\left.\tilde{e}\right|^{n} \\
\left.\tilde{h}\right|^{n}
\end{array}\right],
$$

and the modified curl $\tilde{\mathcal{C}}$ specified in (29). According to [2], stability is assured if the matrix $E_{\mathrm{cn}}$ is positive definite and $F_{\mathrm{cn}}+F_{\mathrm{cn}}^{T}$ is positive semi-definite. The latter is trivially true recalling (7)-(8). Further, the theory exposed in [2, eq. 22-30] shows that $E_{\mathrm{cn}}$ is positive definite if the time step satisfies (43). Rather unexpectedly, losses do not alter the stability limit. 
Both stability analyses of CN implicitization lead to the same conclusion, being that - similar as was the case for Newmark implicitization - proper choices of $\mathcal{I}_{1}$ and $\mathcal{I}_{2}$ (Section 3.2.1) or $\mathcal{P}$ (Section 3.2.2), i.e. well-chosen implicitization, leads to a relaxation of the stability limit.

\subsection{ADI implicitization}

We start this section by presenting a rather atypical stability proof of the traditional leapfrog ADI method, again inspired by [2]. Next, we use the acquired insights to construct a leapfrog ADI formalism that allows implicitization in preferable directions. Finally, the novel leapfrog ADHIE method is used for local implicitization of the conventional FDTD method.

\subsubsection{Stability analysis of leapfrog ADI-FDTD}

The standard ADI method splits the curl (10) into a sum $\mathcal{C}=\mathcal{C}_{1}+\mathcal{C}_{2}$ with the two contributions given by

$$
\begin{aligned}
& \mathcal{C}_{1}=\left[\begin{array}{ccc}
0 & 0 & I_{n_{x}} \otimes \mathcal{D}_{n_{y}} \otimes I_{n_{z}-1} \\
I_{n_{x}-1} \otimes I_{n_{y}} \otimes \mathcal{D}_{n_{z}} & 0 & 0 \\
0 & \mathcal{D}_{n_{x}} \otimes I_{n_{y}-1} \otimes I_{n_{z}} & 0
\end{array}\right] \\
& \mathcal{C}_{2}=\left[\begin{array}{ccc}
0 & -I_{n_{x}} \otimes I_{n_{y}-1} \otimes \mathcal{D}_{n_{z}} & 0 \\
0 & 0 & -\mathcal{D}_{n_{x}} \otimes I_{n_{y}} \otimes I_{n_{z}-1} \\
-I_{n_{x}-1} \otimes \mathcal{D}_{n_{y}} \otimes I_{n_{z}} & 0 & 0
\end{array}\right] .
\end{aligned}
$$

We insert

$$
\begin{array}{rlrl}
\mathcal{C}_{h} & =\hat{\mathcal{V}} \mathcal{C} \hat{\mathcal{W}} & & \mathcal{C}_{e}=\mathcal{V} \mathcal{C}^{T} \mathcal{W} \\
\mathcal{C}_{h 1}=\hat{\mathcal{V}} \mathcal{C}_{1} \hat{\mathcal{W}} & & \mathcal{C}_{e 1}=\mathcal{V} \mathcal{C}_{1}^{T} \mathcal{W} \\
\mathcal{C}_{h 2}=\hat{\mathcal{V}} \mathcal{C}_{2} \hat{\mathcal{W}} & \mathcal{C}_{e 2}=\mathcal{V} \mathcal{C}_{2}^{T} \mathcal{W}
\end{array}
$$

into the update equations (21) without sources and with $\alpha=1$. Next, the symmetry-introducing transformation matrices (28) are used again and both sides of the equation are multiplied by the time step $\Delta t$. This yields

$$
\left.\left(E_{\text {adi }}+F_{\text {adi }}\right) \tilde{x}\right|^{n+1}=\left.\left(E_{\text {adi }}-F_{\text {adi }}\right) \tilde{x}\right|^{n},
$$


with decomposed update matrices

$$
E_{\text {adi }}=\left[\begin{array}{cc}
I_{n_{e}}+\frac{\Delta t^{2}}{4} \tilde{\mathcal{C}}_{1} \tilde{\mathcal{C}}_{1}^{T} & -\frac{\Delta t}{2}\left(\tilde{\mathcal{C}}_{1}+\tilde{\mathcal{C}}_{2}\right) \\
-\frac{\Delta t}{2}\left(\tilde{\mathcal{C}}_{1}+\tilde{\mathcal{C}}_{2}\right)^{T} & I_{n_{h}}+\frac{\Delta t^{2}}{4} \tilde{\mathcal{C}}_{2}^{T} \tilde{\mathcal{C}}_{2}
\end{array}\right] \quad F_{\text {adi }}=\left[\begin{array}{cc}
\frac{\Delta t}{2} D_{\varepsilon}^{-1} D_{\sigma_{e}} & -\frac{\Delta t}{2}\left(\tilde{\mathcal{C}}_{1}+\tilde{\mathcal{C}}_{2}\right) \\
\frac{\Delta t}{2}\left(\tilde{\mathcal{C}}_{1}+\tilde{\mathcal{C}}_{2}\right)^{T} & \frac{\Delta t}{2} D_{\mu}^{-1} D_{\sigma_{m}}
\end{array}\right] .
$$

and rescaled field vector $\left.\tilde{x}\right|^{n}$ specified by (66). The curl parts have undergone the same left- and right-multiplication as the total curl in (29). Since clearly $F_{\text {adi }}+F_{\text {adi }}^{T}$ is positive semi-definite, stability is guaranteed if $E_{\text {adi }}$ is positive definite. Note that $E_{\text {adi }}$ can be factorized into $E_{\text {adi }}=G^{T} G$ with

$$
G=\left[\begin{array}{cc}
I_{n_{e}} & -\frac{\Delta t}{2} \tilde{\mathcal{C}}_{2} \\
-\frac{\Delta t}{2} \tilde{\mathcal{C}}_{1}^{T} & I_{n_{h}}
\end{array}\right]
$$

Consequently,

$$
v^{T} E_{\text {adi }} v=v^{T} G^{T} G v=\|G v\|_{2}^{2} \geq 0
$$

In other words, $E_{\text {adi }}$ is positive semi-definite. In theory, linear instability could still occur for infelicitous time steps which render a singular matrix $E_{\text {adi }}$, corresponding to the pole $z=-1$. Even if this theoretical case exists, in practice, it is very unlikely to exactly pick such an unstable time step within the range of the machine precision. Therefore, as also reported in literature [8], the traditional leapfrog ADI-FDTD method may indeed be considered as unconditionally stable.

\subsubsection{Leapfrog ADHIE-FDTD to implicitize selected dimensions}

Suppose that we want to implicitize the $x$-dimension but not the $y$ - and $z$-dimension, then we propose the following incomplete curl splitting

$$
\begin{aligned}
& \mathcal{C}_{x 1}=\left[\begin{array}{ccc}
0 & 0 & 0 \\
0 & 0 & 0 \\
0 & \mathcal{D}_{n_{x}} \otimes I_{n_{y}-1} \otimes I_{n_{z}} & 0
\end{array}\right] \\
& \mathcal{C}_{x 2}=\left[\begin{array}{ccc}
0 & 0 & 0 \\
0 & 0 & -\mathcal{D}_{n_{x}} \otimes I_{n_{y}} \otimes I_{n_{z}-1} \\
0 & 0 & 0
\end{array}\right] \\
& \mathcal{C}_{y z}=\left[\begin{array}{ccc}
0 & 0
\end{array}\right]
\end{aligned}
$$


such that the total curl is given by $\mathcal{C}=\mathcal{C}_{x 1}+\mathcal{C}_{x 2}+\mathcal{C}_{y z}$. Now, we insert

$$
\begin{array}{rlrl}
\mathcal{C}_{h} & =\hat{\mathcal{V}} \mathcal{C} \hat{\mathcal{W}} & & \mathcal{C}_{e}=\mathcal{V} \mathcal{C}^{T} \mathcal{W} \\
\mathcal{C}_{h 1} & =\hat{\mathcal{V}} \mathcal{C}_{x 1} \hat{\mathcal{W}} & & \mathcal{C}_{e 1}=\mathcal{V} \mathcal{C}_{x 1}^{T} \mathcal{W} \\
\mathcal{C}_{h 2}=\hat{\mathcal{V}} \mathcal{C}_{x 2} \hat{\mathcal{W}} & & \mathcal{C}_{e 2}=\mathcal{V} \mathcal{C}_{x 2}^{T} \mathcal{W}
\end{array}
$$

into (21) and proceed as in Section 3.3.1. The only change to (70), is a modified matrix $E_{\text {adi }}$, given by

$$
\begin{aligned}
E_{\text {adi }} & =\left[\begin{array}{cc}
I_{n_{e}}+\left(\frac{\Delta t}{2 \alpha}\right)^{2} \tilde{\mathcal{C}}_{x 1} \tilde{\mathcal{C}}_{x 1}^{T} & -\frac{\Delta t}{2}\left(\tilde{\mathcal{C}}_{x 1}+\tilde{\mathcal{C}}_{x 2}+\tilde{\mathcal{C}}_{y z}\right) \\
-\frac{\Delta t}{2}\left(\tilde{\mathcal{C}}_{x 1}+\tilde{\mathcal{C}}_{x 2}+\tilde{\mathcal{C}}_{y z}\right)^{T} & I_{n_{h}}+\left(\frac{\Delta t}{2 \alpha}\right)^{2} \tilde{\mathcal{C}}_{x 2}^{T} \tilde{\mathcal{C}}_{x 2}
\end{array}\right] \\
= & \underbrace{\left[\begin{array}{cc}
\alpha I_{n_{e}} & -\frac{\Delta t}{2 \alpha} \tilde{\mathcal{C}}_{x 2} \\
-\frac{\Delta t}{2 \alpha} \tilde{\mathcal{C}}_{x 1}^{T} & \alpha I_{n h}
\end{array}\right]}_{G_{x}^{T}} \underbrace{\left[\begin{array}{cc}
\alpha I_{n_{e}} & -\frac{\Delta t}{2 \alpha} \tilde{\mathcal{C}}_{x 2} \\
-\frac{\Delta t}{2 \alpha} \tilde{\mathcal{C}}_{x 1}^{T} & \alpha I_{n_{h}}
\end{array}\right]}_{G_{x}}+\underbrace{\left[\begin{array}{cc}
\left(1-\alpha^{2}\right) I_{n_{e}} & -\frac{\Delta t}{2} \tilde{\mathcal{C}}_{y z} \\
-\frac{\Delta t}{2} \tilde{\mathcal{C}}_{y z}^{T} & \left(1-\alpha^{2}\right) I_{n h}
\end{array}\right]}_{G_{y z}}
\end{aligned}
$$

Analogous to $G^{T} G$ in Section 3.3.1, $G_{x}^{T} G_{x}$ is positive semi-definite. Consequently, stability is ensured if $G_{y z}$ is positive definite, which, along the lines of [2, eq. 22-30], is found to be the case if

$$
\Delta t<\left(1-\alpha^{2}\right) \frac{2}{\left\|\tilde{\mathcal{C}}_{y z}\right\|_{2}} .
$$

This is the 2D Courant limit in the $y z$-plane reduced by a factor $\left(1-\alpha^{2}\right)$. Based on the conventional leapfrog ADI scheme discussed in Section 3.3.1, the most intuitive value for $\alpha$ would be one. In this case, however, (79) does not yield a valid upper bound for the time step. The scalar $\alpha$ poses a trade-off: the smaller $\alpha$, the larger the maximum time step, but the more splitting error is introduced into the ADHIE scheme. Compared to the splitting error of the traditional split-step and one-step leapfrog ADI schemes [9, eq. 11], the overall splitting error of our ADHIE scheme is heavily reduced due to the increased sparsity of the split curl parts $\mathcal{C}_{e 1}, \mathcal{C}_{h 1}, \mathcal{C}_{e 2}$ and $\mathcal{C}_{h 2}$. Most importantly, (79) shows that all $x$-dependence is eliminated from the stability limit.

\subsubsection{ADHIE local implicitization to relax the stability limit}

Suppose we want to locally refine our 3D grid in the $x$-direction. This implies a tridiagonal solve for $e_{z}$ and $h_{z}$ in the refined region. In contrast to the above, 
the difficulty now is that $\mathcal{C}_{x 1}$ and $\mathcal{C}_{x 2}$ do not encompass the whole $x$-dimension of the grid. Hence, we are dealing with yet another type of curl splitting:

$$
\begin{aligned}
& \mathcal{C}_{x 1}=\left[\begin{array}{ccc}
0 & 0 & 0 \\
0 & 0 & 0 \\
0 & \left(I_{n_{x}-1}-\mathcal{P}_{x}\right) \mathcal{D}_{n_{x}} \otimes I_{n_{y}-1} \otimes I_{n_{z}} & 0
\end{array}\right] \\
& \mathcal{C}_{x 2}=\left[\begin{array}{ccc}
0 & 0 & 0 \\
0 & 0 & -\left(I_{n_{x}-1}-\mathcal{P}_{x}\right) \mathcal{D}_{n_{x}} \otimes I_{n_{y}} \otimes I_{n_{z}-1}
\end{array}\right] \\
& \mathcal{C}_{y z}=\left[\begin{array}{ccc}
0 & 0 & 0
\end{array}\right] \\
& \left.\begin{array}{ccc}
0 & -I_{n_{x}} \otimes I_{n_{y}-1} \otimes \mathcal{D}_{n_{z}} & I_{n_{x}} \otimes \mathcal{D}_{n_{y}} \otimes I_{n_{z}-1} \\
I_{n_{x}-1} \otimes I_{n_{y}} \otimes \mathcal{D}_{n_{z}} & 0 & -\mathcal{P}_{x} \mathcal{D}_{n_{x}} \otimes I_{n_{y}} \otimes I_{n_{z}-1} \\
-I_{n_{x}-1} \otimes \mathcal{D}_{n_{y}} \otimes I_{n_{z}} & \mathcal{P}_{x} \mathcal{D}_{n_{x}} \otimes I_{n_{y}-1} \otimes I_{n_{z}} & 0
\end{array}\right]
\end{aligned}
$$

$\mathcal{P}_{x}$ is the diagonal projection matrix that is zero if the corresponding dual step needs to be eliminated from the stability limit. It is readily observed that stability is again guaranteed if (79) is satisfied, but this time with $\left\|\tilde{\mathcal{C}}_{y z}\right\|_{2}$ derived from (82). Consequently, a proper choice of $\mathcal{P}_{x}$ can lead to a relaxation of the traditional Courant limit without having to implicitize the entire $x$-dimension.

\section{Numerical validation}

Consider the example depicted in Fig. 2a, where a grid with $8 \times 8 \times 8$ cells and main step size $\Delta=2.5 \mathrm{~mm}$ is locally refined in the $x$-dimension by a factor ten as follows:

$$
\begin{aligned}
& \delta_{x}=\Delta \operatorname{diag}\left(1,1, \frac{1}{10}, \frac{1}{10}, \frac{1}{10}, \frac{1}{10}, 1,1\right) \\
& \delta_{y}=\delta_{z}=\Delta \operatorname{diag}(1,1,1,1,1,1,1,1)
\end{aligned}
$$

The dual nodes are placed halfway the primary nodes, i.e.

$$
\begin{aligned}
& \hat{\delta}_{x}=\Delta \operatorname{diag}\left(1, \frac{11}{20}, \frac{1}{10}, \frac{1}{10}, \frac{1}{10}, \frac{11}{20}, 1\right) \\
& \hat{\delta}_{y}=\hat{\delta}_{z}=\Delta \operatorname{diag}(1,1,1,1,1,1,1) .
\end{aligned}
$$

The most delicate case where all energy is trapped inside a PEC cavity filled with vacuum is analyzed. The cavity is excited by randomly initializing one of 
the electric field components to $1 \mathrm{~V} / \mathrm{m}$. Each simulation performs one million iterations and records the discrete energy

$$
\left.q\right|^{n}=\frac{1}{2}\left(\left.\tilde{e}\right|^{n}\right)^{T}\left(\left.\tilde{e}\right|^{n}\right)+\frac{1}{2}\left(\left.\tilde{h}\right|^{n}\right)^{T}\left(\left.\tilde{h}\right|^{n}\right),
$$

with $\tilde{e}$ and $\tilde{h}$ defined in (66), but interpolated to the same time instance. The goal of the proposed implicitization techniques is to arrive at an efficient and flexible update scheme that preserves this energy function when the time step equals the Courant limit of the coarse part of the grid, i.e.

$$
\Delta t_{\text {coarse }}=\frac{\Delta}{c_{0} \sqrt{3}}=4.8145319 \mathrm{ps} \text {. }
$$

\subsection{Fully explicit}

For conventional leapfrog time stepping, the explicit Courant-like limit (56) and the numerically computed norm-based limit (37) are respectively given by

$$
\begin{aligned}
& \Delta t_{\operatorname{expl}}^{\mathrm{fe}}=0.8418616 \mathrm{ps} \\
& \Delta t_{\mathrm{num}}^{\mathrm{fe}}=0.8890071 \mathrm{ps} .
\end{aligned}
$$

As expected, the submultiplicativity (54) used to find (56) gives rise to a $5.6 \%$ smaller maximum time step. Fig. 4 shows the discrete energy (87) normalized to the initial energy $q_{i n}$ for three different time steps close to $\Delta t_{\text {num }}^{\mathrm{fe}}$. Since the energy function is not explicitly conserved from one iteration to the next, as was also observed in e.g. [11], the initial energy $q_{i n}$ does not necessarily equal the average energy. This instantaneous non-conservative behavior can be ascribed to the staggered nature of the Yee cell. For example, due to the staggered grid and the choice of excitation, the initial energy $q_{i n}$ has no magnetic energy contribution, which is physically impossible in the continuum case, but may be true in the discrete case because there does not exist a discrete magnetic field at the location of the excitation. This explains why the initial energy $q_{i n}$ is always somewhat lower than the average energy in our numerical experiments. Fig. 4 confirms that (37) is the exact stability limit for nonuniform grids. Additionally, the poles of the FDTD system are plotted in Fig. 4d. They are numerically 


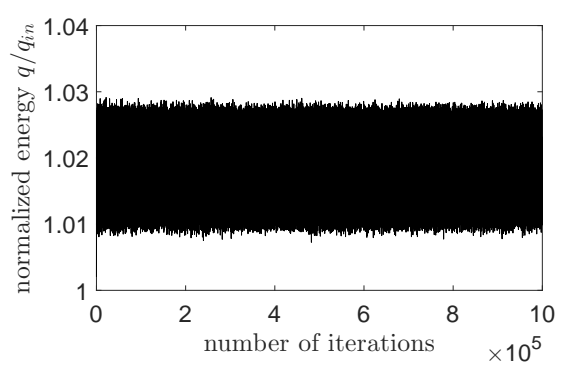

(a)

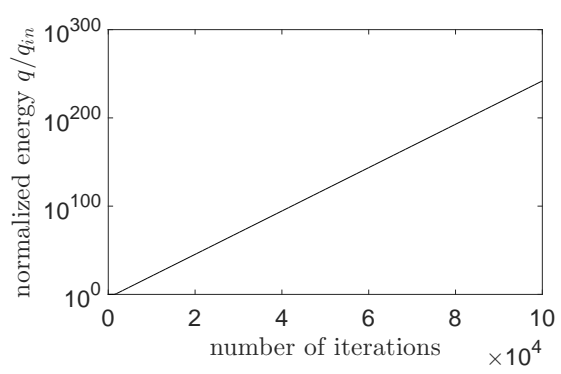

(c)

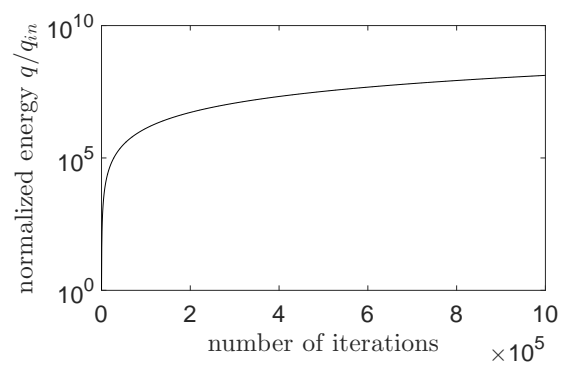

(b)

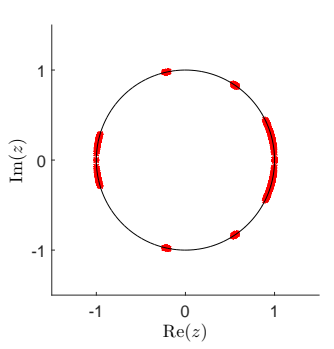

(d)

Figure 4: Normalized discrete energy per iteration for conventional leapfrog time stepping with $\Delta t=(1+\kappa) \Delta t_{\text {num }}^{\mathrm{fe}}$. (a) For $\kappa=-10^{-6}$, the total amount of energy in the cavity is conserved. (b) For $\kappa=0$, the energy increases slower than exponentially. (c) For $\kappa=10^{-6}$, the energy grows exponentially. (d) Location of the poles in the complex plane, which is for the adopted zoom indistinguishable for the three chosen values of $\kappa$.

determined by constructing the amplification matrix and computing its eigenvalues with Matlab's built-in function eig. As expected, all poles of the lossless cavity are located on the unit circle. The number of poles inside the disk with center $z=1$ and a radius of hundred times the machine precision is 854 , which is found to be in exact agreement with (44).

\subsection{Newmark and $C N$ implicitization}

Due to the high degree of resemblance between the Newmark and the CrankNicolson implicitization techniques, they are treated simultaneously in this sec- 
tion. Both techniques use a projection operator of the form (57) eliminating the step sizes of (85) which are smaller than $\Delta$. For our example, $\mathcal{P}_{n_{x}-1}^{i}$ in (57) is given by

$$
\mathcal{P}_{x}=\operatorname{diag}(1,0,0,0,0,0,1) .
$$

Both implicitization techniques have an identical maximum time step specified by (43), which is numerically determined to be

$$
\Delta t_{\text {num }}^{\mathrm{cn}}=5.3562296 \mathrm{ps} .
$$

The late-time behavior of the normalized energy function is studied in Fig. 5 and Fig. 6 for small perturbations of (92) and confirms that this is indeed the exact upper bound on the time step to guarantee Lyapunov stability. The enhanced stability of the simulations running at the exact time step limit (Fig. 5b and Fig. 6b) could be explained by the fact that the fully implicit Newmark (and CN) method cannot have poles $z=-1$, as discussed at the end of Section 3.1.2. Hence, it is likely that for a locally implicitized scheme, the chance of exciting an unstable mode with $z=-1$ is small. As a side note, the spectra of both amplification matrices (Fig. 5d and Fig. 6d) are very similar, as could be expected from our stability analysis in Section 3.2.1. They probably do not exactly coincide due to the numerical error inherent to the eigenvalue computation. Compared to the conventional FDTD method (Fig. 4d), the dynamic poles are pushed towards $z=-1$ due to the smaller time step. All three spectra have the same number of static modes. In conclusion, both implicitization techniques render a stable system for the pursued coarse time step (88).

\subsection{ADI implicitization}

We adopt the split curl stencil (80)-(82) with projection operator (91). For this example, the leapfrog ADHIE update scheme employs $\alpha=0.5$. A numerical estimation of the maximum time step (79) is then given by

$$
\Delta t_{\text {num }}^{\text {adi }}=4.0171722 \mathrm{ps} .
$$




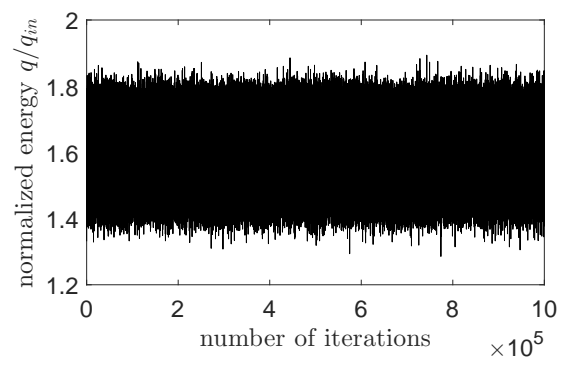

(a)

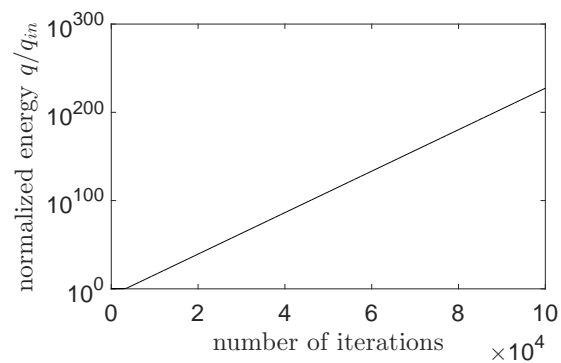

(c)

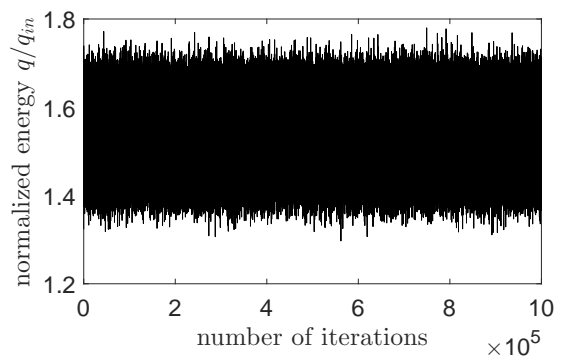

(b)

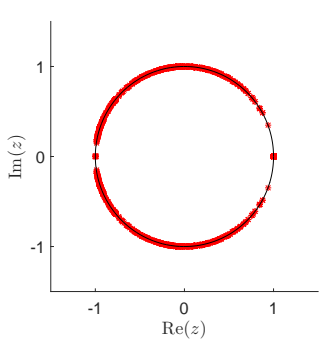

(d)

Figure 5: Normalized discrete energy per iteration for Newmark implicitization with $\Delta t=(1+\kappa) \Delta t_{\mathrm{num}}^{\mathrm{cn}}$. (a) For $\kappa=-10^{-6}$, the total amount of energy in the cavity is conserved. (b) For $\kappa=0$, the energy is still conserved. (c) For $\kappa=10^{-6}$, the energy grows exponentially. (d) Location of the poles in the complex plane, which is for the adopted zoom indistinguishable for the three chosen values of $\kappa$. The non-physical zero pole of the quadratic eigenvalue problem, which has multiplicity $n_{e}+n_{h}$, is omitted. 


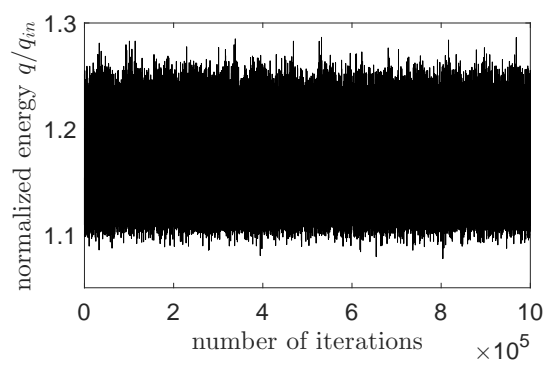

(a)

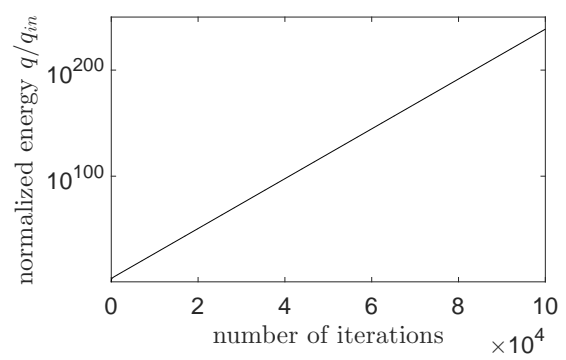

(c)

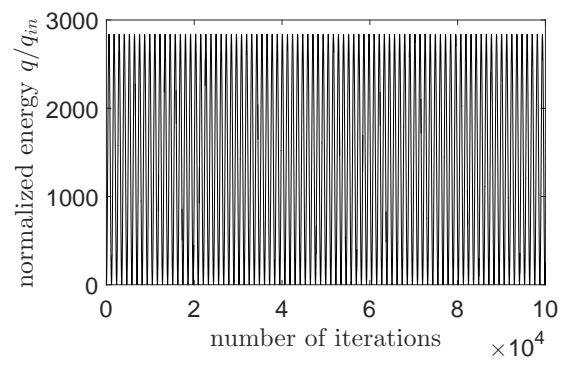

(b)

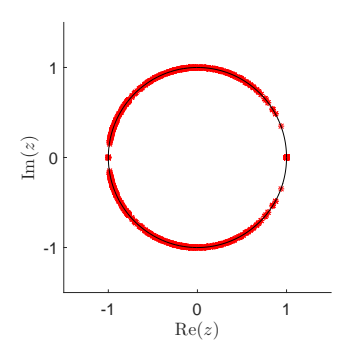

(d)

Figure 6: Normalized discrete energy per iteration for Crank-Nicolson implicitization with $\Delta t=(1+\kappa) \Delta t_{\text {num }}^{\text {cn }}$ (a) For $\kappa=-10^{-6}$, the total amount of energy in the cavity is conserved. (b) For $\kappa=0$, the energy is still conserved but shows large fluctuations, indicating nearly unstable behavior. (c) For $\kappa=10^{-6}$, the energy grows exponentially. (d) Location of the poles in the complex plane, which is for the adopted zoom indistinguishable for the three chosen values of $\kappa$. 


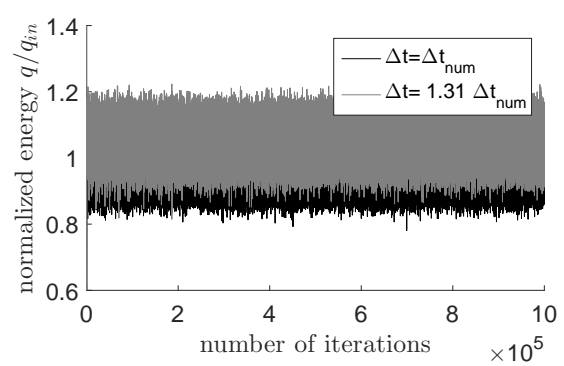

(a)

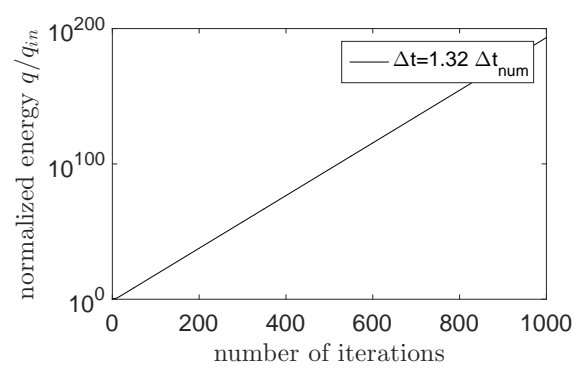

(b)

Figure 7: Normalized discrete energy per iteration for ADI implicitization with different time steps (relative to $\Delta t_{\text {num }}^{\text {adi }}$ ).

The small steps are clearly eliminated from the time step since (93) is five times larger than (90), but unfortunately (93) is smaller than the desired time step (88). Fig. 7 confirms that the local implicitization technique is stable, but also shows that the upper bound (79) is not the exact upper bound. Numerical experiments to determine the actual upper bound reveal that, for this case, the upper bound (93) is underestimated by a factor 1.31. Consequently, the system also remains stable for the desired time step (88). This can be explained as follows. The maximum time step allowed by (79) corresponds to $\lambda_{\min }\left(G_{y z}\right)=0$ but not necessarily to $\lambda_{\min }\left(G_{x}^{T} G_{x}\right)=0$. There is additional margin hidden in the latter, since it is known that $\lambda_{\min }(E) \geq \lambda_{\min }\left(G_{x}^{T} G_{x}\right)+\lambda_{\min }\left(G_{y z}\right)[15$, p. 428].

\section{Conclusion}

The stability of three different local implicitization techniques was rigorously proven for nonuniform tensor product grids with isotropic, inhomogeneous, possibly lossy media enclosed in a PEC box. This resulted in an exact norm-based upper bound for the time step in case of Newmark and CN implicitization, and a more loose upper bound for ADI implicitization. Also, an explicit, Courant-like maximum time step was determined for fully explicit, nonuniform grids. Among the proposed methods, Newmark implicitization is the most computationally expensive technique, as it has a three-level update scheme. The ADI implicitiza- 
tion method is the most efficient one, because, no matter how many dimensions are implicitized, the occurring matrices are tridiagonal and scale with only one dimension. However, it suffers from a splitting error. Fortunately, for the newly proposed leapfrog ADHIE-FDTD method, this splitting error is heavily reduced compared to the traditional (leapfrog) ADI-FDTD method since the split curl parts are more sparse.

Future work includes the extension of the stability analysis to perfectly matched layers (PMLs). By means of a final, short discussion on this matter, it is worth mentioning that our $z$-domain stability analysis is well-suited to analyze the convolutional PML as the discrete convolution is transformed to a simple multiplication in the $z$-domain. Multiplying the step sizes by the $z$-domain stretching factors, our stability analysis can be repeated up to formula (34), but then the $z$-dependence of the step size matrices inside the modified curl operator (29) gives rise to a nonlinear eigenvalue problem with complex (instead of real) symmetry, which strongly impedes further conclusions about the location of $z$ (or $\zeta$ ) in the complex plane.

\section{Appendix}

Lemma 1. If $A, B \in \mathbb{C}^{n \times n}$ are hermitian and $A$ is regular, then there exist $n$ linearly independent vectors $v \in \mathbb{C}^{n \times 1}$ satisfying $A v=\lambda B v$.

Proof. Since the matrices $A$ and $B$ are hermitian, all eigenvalues $\lambda$ reside in $\mathbb{R} \cup\{\infty\}$. We will prove the existence of a full linear independent set of eigenvectors by ruling out the existence of non-trivial Jordan blocks in the Weierstrass canonical form. First, suppose that the finite eigenvalue $\lambda_{r} \in \mathbb{R}$ gives rise to a Jordan block of size $k>1$, then there must exist a Jordan chain [18, p. 4065]

$$
\begin{aligned}
& \left(A-\lambda_{r} B\right) v_{1}=0 \\
& \left(A-\lambda_{r} B\right) v_{i}=B v_{i-1} \quad i=2, \ldots, k .
\end{aligned}
$$

Consequently,

$$
v_{1}^{H} B v_{1}=v_{2}^{H}\left(A-\lambda_{r} B\right)^{H} v_{1}=v_{2}^{H}\left(A-\lambda_{r} B\right) v_{1}=0,
$$


where the first equality holds because of (95) with $i=2$, the second equality uses the hermitian symmetry of the matrix pencil $\left(A-\lambda_{r} B\right)$, and the third equality uses (94). Since $\lambda_{r}$ is finite, $v_{1}$ cannot reside in the null space of $B$. Hence, (96) implies $v_{1}=0$, which is in contradiction with the existence of the Jordan block.

Second, suppose that $\infty$ is an eigenvalue corresponding to a Jordan block of size $k>1$, then there must exist a Jordan chain [18, p. 4065]

$$
\begin{aligned}
& B v_{1}=0 \\
& B v_{i}=A v_{i-1} \quad i=2, \ldots, k
\end{aligned}
$$

Repeating the steps from (96), now yields

$$
v_{1}^{H} A v_{1}=0 .
$$

Since $A$ is regular, we conclude that $v_{1}=0$, which rules out the existence of the Jordan block.

[1] K. Yee, Numerical solution of initial boundary value problems involving Maxwell's equations in isotropic media, IEEE Transactions on Antennas and Propagation 14 (3) (1966) 302-307. doi:10.1109/TAP.1966. 1138693.

[2] B. Denecker, L. Knockaert, F. Olyslager, D. De Zutter, A new state-spacebased algorithm to assess the stability of the finite-difference time-domain method for 3D finite inhomogeneous problems, AEU - International Journal of Electronics and Communications 58 (5) (2004) 339 - 348. doi:10. $1078 / 1434-8411-54100253$.

[3] R. F. Remis, Stability of FDTD on nonuniform grids for Maxwell's equations in lossless media, Journal of Computational Physics 218 (2) (2006) 594 -606. doi:10.1016/j.jcp.2006.02.022.

[4] R. A. Chilton, R. Lee, The discrete origin of FETD-Newmark late time instability, and a correction scheme, Journal of Computational Physics 224 (2) (2007) 1293 - 1306. doi:10.1016/j.jcp.2006.11.021. 
[5] S. B. Shi, W. Shao, X. K. Wei, X. S. Yang, B. Z. Wang, A new unconditionally stable FDTD method based on the Newmark-beta algorithm, IEEE Transactions on Microwave Theory and Techniques 64 (12) (2016) 4082-4090. doi:10.1109/TMTT.2016.2608340.

[6] B. De Deckere, A. Van Londersele, D. De Zutter, D. Vande Ginste, Birefringent dispersive FDTD subgridding scheme, Electronics Letters 52 (17) (2016) 1455-1457. doi:10.1049/el.2016.1709.

[7] C. Sun, C. W. Trueman, Unconditionally stable Crank-Nicolson scheme for solving two-dimensional Maxwell's equations, Electronics Letters 39 (7) (2003) 595-597. doi:10.1049/el:20030416.

[8] S. C. Yang, Z. Chen, Y. Yu, W. Y. Yin, An unconditionally stable one-step arbitrary-order leapfrog ADI-FDTD method and its numerical properties, IEEE Transactions on Antennas and Propagation 60 (4) (2012) 1995-2003. doi:10.1109/TAP.2012.2186249.

[9] A. Grande, J. A. Pereda, Accuracy limitations of the locally onedimensional FDTD technique, IEEE Antennas and Wireless Propagation Letters 13 (2014) 1180-1183. doi:10.1109/LAWP.2014.2330761.

[10] K. Abdijalilov, H. Grebel, Z-transform theory and FDTD stability, IEEE Transactions on Antennas and Propagation 52 (11) (2004) 2950-2954. doi:10.1109/TAP.2004.835267.

[11] R. A. Chilton, R. Lee, Conservative and provably stable FDTD subgridding, IEEE Transactions on Antennas and Propagation 55 (9) (2007) 25372549. doi:10.1109/TAP.2007.904092.

[12] T. Rylander, A. Bondeson, Stability of explicit-implicit hybrid timestepping schemes for Maxwell's equations, Journal of Computational Physics 179 (2) (2002) 426-438. doi:10.1006/jcph.2002.7063.

[13] G. Strang, Introduction to Linear Algebra (4th edition), Wellesley - Cambridge Press, 2009. 
[14] S. Wang, F. L. Teixeira, Some remarks on the stability of time-domain electromagnetic simulations, IEEE Transactions on Antennas and Propagation 52 (3) (2004) 895-898. doi:10.1109/TAP.2004.825486.

[15] D. Bernstein, Matrix Mathematics (2nd edition), Princeton University Press, 2009.

[16] R. F. Remis, On the stability of the Finite-Difference Time-Domain method, Journal of Computational Physics 163 (1) (2000) 249 - 261. doi:http://dx.doi.org/10.1006/jcph.2000.6573.

[17] M. Min, C. Teng, The instability of the Yee scheme for the magic time step, Journal of Computational Physics 166 (2) (2001) 418 - 424. doi: http://dx.doi.org/10.1006/jcph.2000.6650.

[18] T. Berger, A. Ilchmann, S. Trenn, The quasi-Weierstrass form for regular matrix pencils, Linear Algebra and its Applications 436 (10) (2012) 4052 4069. doi:http://dx.doi.org/10.1016/j.laa.2009.12.036. 